ing the origin, the theorem from Polya-Szegö may be applied with the $F(z)$ of the theorem taken as $A(z)$. Theorem III(b) then follows immediately.

As an application of Theorem III, let us consider the polynomial $F(z)=\sum_{k=0}^{m} a_{k} G(k+p) z^{k}$ where $p>0$ and $G(z)=\Gamma(z)^{-1}$ $=e^{\mu z} \prod_{n=1}^{\infty}\left(1+n^{-1} z\right) e^{-z / n}$, the reciprocal of the gamma function. Since $\nu=0$ and all the zeros of $G(z+p)$ are negative, any sector $\omega_{1} \leqq \arg z \leqq \omega_{2} \leqq \pi-\omega_{1}$ containing all the zeros of $A(z)$ will also contain all the zeros of $F(z)$. For example, if $A(z)=(z-2)(z+1-i)$, then $F(z)=0.5 z^{2}-(1+i) z-2+2 i$, which has the zeros $(3.058+0.514 i)$ and $(-1.058+1.486 i)$, both thus being in the sector $0 \leqq \arg z \leqq 135^{\circ}$ containing the zeros of $A(z)$.

University of Wisconsin at Milwaukee

\title{
ON THE EXTENSION OF A VECTOR FUNCTION SO AS TO PRESERVE A LIPSCHITZ CONDITION
}

F. A. VALENTINE

1. Introduction. Let $V$ be a two-dimensional Euclidean space, and let $x$ be a vector ranging over $V$. The vector function $f(x)$ is to be a vector in $V$ defined over a set $S$ of the space $V$. The Euclidean distance between any two points $x$ and $y$ in the plane is denoted by $|x-y|$. Furthermore $f(x)$ is to satisfy a Lipschitz condition, so that there exists a positive constant $K$ such that

$$
\left|f\left(x_{1}\right)-f\left(x_{2}\right)\right| \leqq K\left|x_{1}-x_{2}\right|
$$

holds for all pairs $x_{1}$ and $x_{2}$ in $S$.

In event $f(x)$ is a real-valued function of a variable $x$ ranging over a set $S$ of a metric space, then the extension of the definition of $f(x)$ to any set $T \supset S$ so as to satisfy the condition (1) has been accomplished. ${ }^{1}$ The present paper establishes the result that the vector function $f(x)$ can be extended to any set $T \supset S$ so as to satisfy the Lipschitz condition with the same constant $K$. In $\$ 3$ it is shown how the method used to obtain the above result can be applied to yield an extension for the case considered by McShane. ${ }^{2}$ If $f(x)$ has its

Presented to the Society, April 11, 1942; received by the editors May 11, 1942.

${ }^{1}$ E. J. McShane, Extension of range of functions, Bull. Amer. Math. Soc. vol. 40 (1934) pp. 837-842.

${ }^{2}$ Loc. cit. 
functional values in a metric space then, in general, it cannot be extended so as to preserve (1). The geometric theorems which arise in forming the extension prove to be of as much interest as the extension itself. In the following section it is shown that this extension is a consequence of the following theorem about sets of circles in the plane. A circle with center $x_{i}$ and radius $r_{i}$ is the two-dimensional set of points $x$ for which $\left|x-x_{i}\right| \leqq r_{i}$.

THEOREM 1. Consider in the plane two sets of circles $M$ and $M^{\prime}$ such that to each circle in $M$ there corresponds a circle in $M^{\prime}$. Let $C_{i}^{\prime} \in M^{\prime}$, having center $x_{i}^{\prime}$ and radius $r_{i}^{\prime}$, correspond to $C_{i} \in M$, having center $y_{i}$ and radius $r_{i}$.

Suppose that

$$
r_{i}^{\prime}=r_{i}
$$

for all corresponding circles $C_{i}$ and $C_{i}^{\prime}$. Furthermore suppose that

$$
\left|x_{i}^{\prime}-x_{j}^{\prime}\right| \leqq\left|y_{i}-y_{j}\right|
$$

for all corresponding pairs of circles $\left(C_{i}, C_{j}\right)$ and $\left(C_{i}^{\prime}, C_{j}^{\prime}\right)$. Finally suppose that the intersection of all the circles in $M$ is not empty, so that the product

$$
\prod_{M} C_{i} \neq 0
$$

Then it is true that the intersection of all the circles in $M^{\prime}$ is such that

$$
\prod_{M^{\prime}} C_{i}^{\prime} \neq 0
$$

2. The extension. If the set $S$ consists of a single point $x_{1}$, the extension of $f(x)$ to a second point $x_{2}$ is trivial. We will let $S^{\prime}$ be the set in the $f$-plane which corresponds to the set $S$ in the $x$-plane.

Suppose that the set $S$ consists of two points $x_{1}$ and $x_{2}$, and that we wish to extend $f(x)$ to a third point $x_{3}$. With the points $K x_{j}$ as centers $(j=1,2)$ draw circles $C_{j}$ with radii $r_{j}=K\left|x_{j}-x_{3}\right|$, respectively. Define $x_{j}^{\prime}$ to be

$$
x_{j}^{\prime} \equiv f\left(x_{j}\right), \quad j=1,2 .
$$

With $x_{j}^{\prime}$ as centers draw circles $C_{j}^{\prime}$ with radii $r_{j}^{\prime}=r_{j}$, respectively. Since by hypothesis $\left|x_{1}^{\prime}-x_{2}^{\prime}\right| \leqq K\left|x_{1}-x_{2}\right|$, and since by construction the product $C_{1} \cdot C_{2} \neq 0$, it is true that $C_{1}^{\prime} \cdot C_{2}^{\prime} \neq 0$. To extend $f(x)$ to $x_{3}$ so as to satisfy (1), choose $x_{3}^{\prime} \in C_{1}^{\prime} \cdot C_{2}^{\prime}$. Since $\left|x_{j}^{\prime}-x_{3}^{\prime}\right| \leqq r_{j}^{\prime}=r_{j}$ $=K\left|x_{j}-x_{3}\right|$, letting $f\left(x_{3}\right) \equiv x_{3}^{\prime}$, the function $f(x)$ has been extended from $S=\left(x_{1}, x_{2}\right)$ to $T=\left(x_{1}, x_{2}, x_{3}\right)$ so as to satisfy the Lipschitz condition (1). 
The crucial extension, as we shall see, is from $S=\left(x_{1}, x_{2}, x_{3}\right)$ to $T=\left(x_{1}, x_{2}, x_{3}, x_{4}\right)$. In order to construct this extension, the following lemma and theorem are essential. For convenience denote the triangular set determined by three points $y_{1}, y_{2}, y_{3}$ by $\Delta\left(y_{1}, y_{2}, y_{3}\right)$.

LEMMA 1. If three circles $C_{i}^{\prime}(i=1,2,3)$ with centers $x_{i}^{\prime}$, respectively, are such that

$$
\begin{aligned}
C_{i}^{\prime} \cdot C_{j}^{\prime} \cdot \Delta\left(x_{1}^{\prime}, x_{2}^{\prime}, x_{3}^{\prime}\right) & \neq 0, \quad i, j=1,2,3, \\
C_{1}^{\prime} \cdot C_{2}^{\prime} \cdot C_{3}^{\prime} \cdot \Delta\left(x_{1}^{\prime}, x_{2}^{\prime}, x_{3}^{\prime}\right) & =0,
\end{aligned}
$$

then it is true that ${ }^{3}$

$$
B\left(C_{i}^{\prime}\right) \cdot B\left(C_{j}^{\prime}\right) \cdot \Delta\left(x_{1}^{\prime}, x_{2}^{\prime}, x_{3}^{\prime}\right) \neq 0, \quad i, j=1,2,3,
$$

where $B\left(C_{i}^{\prime}\right)$ is the boundary of the circle $C_{i}^{\prime}$.

To prove Lemma 1 select three points $x_{i j} \in C_{i}^{\prime} \cdot C_{j}^{\prime} \cdot \Delta(i=1,2$; $j=2,3 ; j>i)$ which exist by virtue of (6). Since $\Delta\left(x_{12}^{\prime}, x_{13}^{\prime}, x_{23}^{\prime}\right)$ $C \Delta\left(x_{1}^{\prime}, x_{2}^{\prime}, x_{3}^{\prime}\right)$, in proving (8) it is sufficient to prove

$$
B\left(C_{i}^{\prime}\right) \cdot B\left(C_{j}^{\prime}\right) \cdot \Delta\left(x_{12}^{\prime}, x_{13}^{\prime}, x_{23}^{\prime}\right) \neq 0
$$

Denote the line segment joining points $x$ and $y$ by $\overline{x y}$. Since $C_{1}^{\prime}$ is convex, $\overline{x_{12}^{\prime} x_{13}^{\prime}} \subset C_{1}^{\prime}$.. Also (7) implies that the point $x_{23}^{\prime}$ is not contained in $C_{1}^{\prime}$. Hence the circle $C_{1}^{\prime}$ contains a maximum subsegment $\overline{x_{12}^{\prime} P_{2}}$ of $\overline{x_{12}^{\prime} x_{23}^{\prime}}$ and a maximum subsegment $\overline{x_{13}^{\prime} P_{3}}$ of $\overline{x_{13}^{\prime} x_{23}^{\prime}}$, where the points $P_{2} \neq P_{3} \neq x_{23}^{\prime}$. Since $\overline{P_{2} P_{3}} \subset C_{1}^{\prime}$, since $x_{23}^{\prime} \notin C_{1}^{\prime}$, and since $B\left(C_{1}^{\prime}\right)$ cannot intersect the $B\left[\Delta\left(P_{2}, P_{3}, x_{23}^{\prime}\right)\right]$ except at $P_{2}$ and $P_{3}$, a simple arc of $B\left(C_{1}^{\prime}\right)$ joins $P_{2}$ and $P_{3}$ and is contained in $\Delta\left(P_{2}, P_{3}, x_{23}^{\prime}\right)$ and hence in $\Delta\left(x_{12}^{\prime}, x_{13}^{\prime}, x_{23}^{\prime}\right)$.

In exactly the same way, there exist points $Q_{3} \in \overline{x_{13}^{\prime} x_{23}^{\prime}}, Q_{1} \in \overline{x_{12}^{\prime} x_{13}^{\prime}}$, with $Q_{1} \neq Q_{3} \neq x_{13}^{\prime}$, such that $Q_{1}$ and $Q_{3}$ are joined by a simple arc of $B\left(C_{2}^{\prime}\right)$ contained in $\Delta\left(x_{12}^{\prime}, x_{13}^{\prime}, x_{23}^{\prime}\right)$. Suppose the point $P_{3}$ lay be-

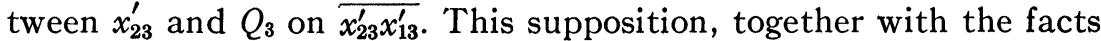
$C_{2}^{\prime} \supset \overline{x_{23}^{\prime} Q_{3}}, \quad C_{1}^{\prime} \supset \overline{x_{13}^{\prime} P_{3}}$, implies that $\overline{x_{23}^{\prime} x_{13}^{\prime}} \subset C_{1}^{\prime}+C_{2}^{\prime}$. But since $\overline{x_{23}^{\prime} x_{13}^{\prime}} \subset C_{3}^{\prime}$, the previous statement implies that $C_{1}^{\prime} \cdot C_{2}^{\prime} \cdot C_{3}^{\prime} \cdot \Delta \neq 0$, contrary to assumption (7). Hence $P_{3}$ is not between $x_{23}^{\prime}$ and $Q_{3}$ on

${ }^{3}$ Lemma 1 is stated for circles in order to shorten the proof of Theorem 2; however the lemma can be generalized as follows: Suppose that each of the sets $C_{1}, C_{2}, C_{3}, \Delta$ and each of the products $C_{i} \cdot \Delta, C_{i} \cdot C_{i} \cdot \Delta(i, j=1,2,3)$ is a simply connected plane set having a simple closed curve as its boundary. Furthermore suppose $C_{1} \cdot C_{2} \cdot C_{3}=0$, $C_{i} \cdot C_{j} \cdot B(\Delta) \neq 0$, where $B(\Delta)$ is the boundary of $\Delta$. Then it is true that $B\left(C_{i}\right) \cdot B\left(C_{j}\right) \cdot \Delta$ $\neq 0$. 
$\overline{x_{23}^{\prime} x_{13}^{\prime}}$, so that $Q_{3} \in \overline{x_{23}^{\prime} x_{13}^{\prime}}-\overline{x_{13}^{\prime} P_{3}}$. This implies that the points $P_{2}, Q_{3}$, $P_{3}, Q_{1}$ lie on the boundary of $\Delta\left(x_{12}^{\prime}, x_{13}^{\prime}, x_{23}^{\prime}\right)$ in a clockwise order in the order just given.

Since $P_{2}$ and $P_{3}$ are joined by a simple arc of $B\left(C_{1}^{\prime}\right)$ contained in $\Delta\left(x_{12}^{\prime}, x_{13}^{\prime}, x_{23}^{\prime}\right)$, and since $Q_{1}$ and $Q_{3}$ are joined by an arc of $B\left(C_{2}^{\prime}\right)$ having the same properties, condition (9) holds with $i=1, j=2$. Since we can choose any pair of the circles $C_{i}^{\prime}$ and repeat the above argument, condition (9) has been proved, and hence Lemma 1 is established.

THEOREM 2. Suppose the two triangles $\Delta\left(y_{1}, y_{2}, y_{3}\right)$ and $\Delta\left(x_{1}^{\prime}, x_{2}^{\prime}, x_{3}^{\prime}\right)$ are such that

$$
\left|x_{i}^{\prime}-x_{j}^{\prime}\right| \leqq\left|y_{i}-y_{j}\right|, \quad i, j=1,2,3 .
$$

Then to any point $y_{4}$ in the plane there corresponds a point $x_{4}^{\prime}$ contained in $\Delta\left(x_{1}^{\prime}, x_{2}^{\prime}, x_{3}^{\prime}\right)$ such that

$$
\left|x_{4}^{\prime}-x_{i}^{\prime}\right| \leqq\left|y_{4}-y_{i}\right|, \quad i=1,2,3 .
$$

To prove Theorem 2 , with $y_{i}$ as centers draw circles $C_{i}$ with radii $r_{i} \equiv\left|y_{4}-y_{i}\right|$, respectively. Similarly with $x_{i}^{\prime}$ as centers draw circles $C_{i}^{\prime}$ with radii $r_{i}^{\prime}=r_{i}$, respectively. Since $y_{4} \in C_{i}$, the products $C_{i} \cdot C_{j} \cdot \Delta\left(y_{1}, y_{2}, y_{3}\right) \neq 0$. Hence condition (10), together with the fact $r_{i}^{\prime}=r_{i}$, implies condition (6).

We first prove that condition (10) implies $C_{1}^{\prime} \cdot C_{2}^{\prime} \cdot C_{3}^{\prime} \cdot \Delta\left(x_{1}^{\prime}, x_{2}^{\prime}, x_{3}^{\prime}\right)$ $\neq 0$. Suppose this were not so. Let $P_{i j}$ be a point of intersection of $B\left(C_{i}^{\prime}\right)$ and $B\left(C_{j}^{\prime}\right)$. Since we suppose (7) holds, Lemma 1 implies that $P_{i j}$ can be chosen so that $P_{i j} \in \Delta\left(x_{1}^{\prime}, x_{2}^{\prime}, x_{3}^{\prime}\right)(i=1,2 ; j=2,3 ; j>i)$. Since we supposed $C_{1}^{\prime} \cdot C_{2}^{\prime} \cdot C_{3}^{\prime} \cdot \Delta=0$, we have

$$
\left|x_{2}^{\prime}-P_{12}\right|<\left|x_{2}^{\prime}-P_{13}\right| \text {. }
$$

Denote the angle less than or equal to $\pi$, determined by three points $a_{1}, a_{2}, a_{3}$, with vertex at $a_{2}$, by $\angle a_{1} a_{2} a_{3}$. Since by definition $\left|x_{1}^{\prime}-P_{12}\right|=\left|x_{1}^{\prime}-P_{13}\right|=r_{1}$, condition (12) implies by the law of cosines that

$$
\angle x_{2}^{\prime} x_{1}^{\prime} P_{12}<\angle x_{2}^{\prime} x_{1}^{\prime} P_{13} \text {. }
$$

Condition (13), together with the fact $P_{13} \in \Delta\left(x_{1}^{\prime}, x_{2}^{\prime}, x_{3}^{\prime}\right)$, implies the first of the inequalities

$$
\begin{aligned}
& \angle x_{2}^{\prime} x_{1}^{\prime} P_{12}+\angle x_{3}^{\prime} x_{1}^{\prime} P_{13}<\angle x_{2}^{\prime} x_{1}^{\prime} x_{3}^{\prime}, \\
& \angle x_{1}^{\prime} x_{3}^{\prime} P_{13}+\angle x_{2}^{\prime} x_{3}^{\prime} P_{23}<\angle x_{1}^{\prime} x_{3}^{\prime} x_{2}^{\prime}, \\
& \angle x_{3}^{\prime} x_{2}^{\prime} P_{23}+\angle x_{1}^{\prime} x_{2}^{\prime} P_{12}<\angle x_{3}^{\prime} x_{2}^{\prime} x_{1}^{\prime} .
\end{aligned}
$$


The last two inequalities are proved exactly as the first.

The sum of all the angles in the three triangles $\Delta\left(x_{i}^{\prime}, P_{i j}, x_{j}^{\prime}\right)$ $(i=1,2 ; j=2,3 ; j>i)$ is $3 \pi$. By adding the inequalities in (14), we see that in these three triangles $\Delta\left(x_{i}^{\prime}, P_{i j}, x_{j}^{\prime}\right)$ the sum of those six angles, each of which has its vertex at one of the points $x_{1}^{\prime}, x_{2}^{\prime}, x_{3}^{\prime}$ is less than $\pi$. Hence the sum of the remaining three angles in $\Delta\left(x_{i}^{\prime}, P_{i j}, x_{j}^{\prime}\right)$ satisfies the condition

$$
\sum_{i, j} \angle x_{i}^{\prime} P_{i j} x_{j}^{\prime}>2 \pi, \quad i=1,2 ; j=2,3 ; j>i .
$$

Furthermore for the $\Delta\left(y_{1}, y_{2}, y_{3}\right)$ of Theorem 2 we have

$$
\sum_{i, j} \angle y_{i} y_{4} y_{j} \leqq 2 \pi, \quad i=1,2 ; j=2,3 ; j>i,
$$

the equality holding if $y_{4} \in \Delta\left(y_{1}, y_{2}, y_{3}\right)$; otherwise the inequality holds. Consequently at least one of the three angles in (15) is greater than the corresponding angle in (16). Without loss of generality renumber the angles so that

$$
\angle x_{1}^{\prime} P_{12} x_{2}^{\prime}>\angle y_{1} y_{4} y_{2} \text {. }
$$

By construction $\left|x_{i}^{\prime}-P_{12}\right|=\left|y_{i}-y_{4}\right|(i=1,2)$. Condition (17) implies by the law of cosines that in the triangles $\Delta\left(x_{1}^{\prime}, P_{12}, x_{2}^{\prime}\right)$ and $\Delta\left(y_{1}^{\prime}, y_{4}^{\prime}, y_{2}\right)$

$$
\left|x_{1}^{\prime}-x_{2}^{\prime}\right|>\left|y_{2}-y_{1}\right| \text {. }
$$

This contradicts assumption (10). Hence the assumption that (7) holds is false. Thus choosing a point $x_{4}^{\prime} \in C_{1}^{\prime} \cdot C_{2}^{\prime} \cdot C_{3}^{\prime} \cdot \Delta \neq 0$, we have $\left|x_{4}^{\prime}-x_{i}^{\prime}\right| \leqq r_{i}^{\prime}=r_{i}=\left|y_{i}-y_{4}\right|(i=1,2,3)$ and Theorem 2 has been established.

We now readily establish the following theorem.

THEOREM 3. If the plane vector $f(x)$ is defined on a plane set $S=\left(x_{1}, x_{2}, x_{3}\right)$ so as to satisfy the Lipschitz condition (1), then it can be extended to any plane set $T=\left(x_{1}, x_{2}, x_{3}, x_{4}\right)$ so as to be Lipschitz preserving. The extension $f\left(x_{4}\right)$ can be defined so as to be contained in the triangle formed by the points $f\left(x_{i}\right)(i=1,2,3)$.

Proof. In Theorem 2, let

$$
y_{i} \equiv K x_{i}, \quad x_{i}^{\prime} \equiv f\left(x_{i}\right), \quad y_{4} \equiv K x_{4}, \quad i=1,2,3 .
$$

Then the point $x_{4}^{\prime}$ in Theorem 2 is the desired extension $f\left(x_{4}\right) \equiv x_{4}^{\prime}$, since conditions (10) and (11) imply that

$$
\left|f\left(x_{j}\right)-f\left(x_{k}\right)\right| \leqq K\left|x_{j}-x_{k}\right|, \quad j, k=1, \cdots, 4 .
$$


In order to extend $f(x)$ from an arbitrary set $S$ to any set $T \supset S$ we first prove Theorem 1 . This can be accomplished by means of Theorem 2 and by a theorem of Helly ${ }^{4}$ as follows. Choose an arbitrary but fixed set of three circles $C_{i}(i=1,2,3)$ in the set of circles $M$ of Theorem 1. Since in Theorem 1 the hypotheses include the fact that $\prod_{M} C_{i} \neq 0$, choose the arbitrary point $y_{4}$ in Theorem 2 so that $y_{4} \in \prod_{i=1}^{3} C_{i}$. Then Theorem 2 implies that there exists a point $x_{4}^{\prime}$ such that $\left|x_{4}^{\prime}-x_{i}^{\prime}\right| \leqq\left|y_{4}-y_{i}\right|$. Since $y_{4} \in \prod_{i=1}^{3} C_{i}$, it is true that $\left|y_{4}-y_{i}\right| \leqq r_{i}$, whence $\left|x_{4}^{\prime}-x_{i}^{\prime}\right| \leqq r_{i}$. Since $r_{i}^{\prime}=r_{i}$ in Theorem 1 , the above inequalities imply that each set of three circles in $M^{\prime}$ has a point common to all three circles. Now by Helly's theorem, with $n=2$, it is true that all the circles in $M^{\prime}$ have a point in common. Thus Theorem 1 is established.

Now we are in a position to prove the following theorem.

THEOREM 4. Suppose the plane vector function $f(x)$ is defined and satisfies the Lipschitz condition (1) on a set $S$ of the plane.

Then if $T$ is any set containing $S$, it is true that $f(x)$ can be extended to $T$ so as to preserve the Lipschitz condition (1). The extension of $f(x)$ can be defined so that the set of points $(f(x)$, for $x \in T)$ is contained in any prescribed closed convex set containing $S^{\prime}$, where $S^{\prime} \equiv(f(x)$, for $x \in S)$.

ProOF. First, we prove that if $U$ is any set on which $f(x)$ satisfies the Lipschitz condition (1), and if $x_{0}$ is an arbitrary point exterior to $U$, then $f(x)$ can be extended from $U$ to $U+x_{0}$ so as to satisfy (1). To do this let $x_{i}$ be an arbitrary point in $U$, and let

$$
x_{i}^{\prime} \equiv f\left(x_{i}\right)
$$

be the corresponding point in $U^{\prime} \equiv[f(x), x \in U]$. With $y_{i}=K x_{i}$ as center and with radius $r_{i} \equiv K\left|x_{0}-x_{i}\right|$ draw a circle $C_{i}$. As $x_{i}$ ranges over $U$, denote the set of circles thus defined by $M$. Similarly with $x_{i}^{\prime}$ as center and with radius $r_{i}^{\prime}=r_{i}$ draw a circle $C_{i}^{\prime}$, and denote the set of all circles, as $x_{i}^{\prime}$ ranges over $U^{\prime}$, by $M^{\prime}$. Since condition (1) holds on $U$, condition (2) in Theorem 1 holds on $U$. Furthermore since by construction $r_{i}=K\left|x_{0}-x_{i}\right|$ we have $\prod_{M} C_{i} \neq 0$. Hence Theorem 1 implies that $\prod_{M^{\prime}} C_{i}^{\prime} \neq 0$. Consequently there exists a point $x_{0}^{\prime}$, with $x_{0}^{\prime} \in \prod_{M}, C_{i}^{\prime}$, which implies that

$$
\left|x_{0}^{\prime}-x_{i}^{\prime}\right| \leqq r_{i} \equiv K\left|x_{0}-x_{i}\right|
$$

\footnotetext{
${ }^{4}$ E. Helly, Jber. Deutschen Math. Verein vol. 32 (1923) pp. 175-176. The theorem states: If each $n+1$ sets of a family of closed, bounded, convex sets of the n-dimensional Euclidean space intersect, then there is a point common to all the sets.
} 
for all corresponding pairs $x_{i}$ and $x_{i}^{\prime}$ in $U$ and $U^{\prime}$, respectively. Hence letting $f\left(x_{0}\right) \equiv x_{0}^{\prime}$, we have extended $f(x)$ from $U$ to $U+x_{0}$ so that the Lipschitz condition (1) is preserved. For convenience denote the above extension from an arbitrary set $U$ to the set $U+x_{0}$, where $x_{0}$ is arbitrary, as extension $\mathrm{E}$.

The extension from a set $S$ to a region $T \supset S$ of Theorem 4 can be accomplished as follows. Let $R$ be the set of all the points in the plane which have rational coordinates. Extension $\mathrm{E}$ of the preceding paragraph implies by ordinary induction that $f(x)$ can be extended from the set $S$ to the set $S+R$ so as to satisfy the Lipschitz condition (1). Let $\bar{x}$ be any other point in the plane. In order to effect the extension to $\bar{x}$ let $x_{i}$ be any sequence of points with rational coordinates such that $\lim _{i=\infty} x_{i}=\bar{x}$. If we define $U \equiv S+\sum x_{i}, x_{0} \equiv \bar{x}$, then extension $\mathrm{E}$ of the preceding paragraph yields an extension $f(\bar{x})$ so that $f(x)$ satisfies (1) on $U+x_{0}$. Condition (1) implies that $\lim _{i=\infty} f\left(x_{i}\right)=f(\bar{x})$. The function $f(x)$ is defined uniquely at $\bar{x}$, for let $y_{i}$ be any other sequence of rational points such that $\lim _{i=\infty} y_{i}=\bar{x}$. Letting $U \equiv S+\sum y_{i}$, and $x_{0} \equiv \bar{x}$, extension E yields an extension $f^{*}(\bar{x})$ such that $f(x)$ satisfies (1) on $U+x_{0}$. Since

$$
\left|f\left(y_{i}\right)-f\left(x_{i}\right)\right| \leqq K\left|y_{i}-x_{i}\right|,
$$

and since $\lim _{i=\infty} f\left(x_{i}\right)=f(\bar{x})$, it follows that

$$
\lim _{i=\infty} f\left(y_{i}\right)=\lim _{i=\infty} f\left(x_{i}\right)=f^{*}(\bar{x})=f(\bar{x}) .
$$

The function $f(x)$ thus defined satisfies the Lipschitz condition (1) on the whole plane. To prove this statement, let $x$ and $y$ be any two points in the plane, and let $x_{i}$ and $y_{i}$ be two sequences of rational points such that $\lim _{i=\infty} x_{i}=x, \lim _{i=\infty} y_{i}=y$. Then the inequalities

$$
\begin{aligned}
|f(x)-f(y)| & \leqq\left|f(x)-f\left(x_{i}\right)\right|+\left|f\left(x_{i}\right)-f\left(y_{i}\right)\right|+\left|f\left(y_{i}\right)-f(y)\right| \\
& \leqq K\left|x-x_{i}\right|+K\left|x_{i}-y_{i}\right|+K\left|y_{i}-y\right|
\end{aligned}
$$

imply, by passage to the limit, that

$$
|f(x)-f(y)| \leqq K|x-y| .
$$

Thus the first part of Theorem 4 is proved since if we can extend $f(x)$ to the whole plane we can surely extend it to $T \supset S$.

To prove the last sentence of Theorem 4 , let $L$ be any closed convex set such that $L \supset S^{\prime}$. Consider the sets of circles $M$ and $M^{\prime}$ defined in the first paragraph following Theorem 4, however with $U=S$ and $U^{\prime}=S^{\prime}$. Consider any two circles $C_{1}^{\prime}$ and $C_{2}^{\prime}$ in $M^{\prime}$. Since the straight line joining the centers of these circles is in $L$, and since (1) implies 
that $C_{1}^{\prime} \cdot C_{2}^{\prime} \neq 0$, we have $C_{1}^{\prime} \cdot C_{2}^{\prime} \cdot L \neq 0$. Furthermore Theorem $1 \mathrm{im}$ plies that $\prod_{M^{\prime}} C_{i}^{\prime} \neq 0$. Hence the theorem of Helly implies that the product

$$
L \cdot \prod_{M^{\prime}} C_{i}^{\prime} \neq 0 \text {. }
$$

Hence in extension $E$ one can choose $x_{0}^{\prime} \in L$, whence $S^{\prime}+x_{0}^{\prime} \subset L$. Now by ordinary induction the function $f(x)$ can be extended to the set $S+R$ (where $R$ is the set of points with rational coordinates), so that the set $S^{\prime}+R^{\prime} \equiv[f(x), x$ ranges over $S+R] \subset L$. Now the extension of $f(x)$ to the whole plane so that $f(x) \subset L, x$ arbitrary, can be constructed. For consider the point $\bar{x}$ in equation (19), where $x_{i} \subset R$. Since by (19) $\lim _{i=\infty} f\left(x_{i}\right)=f(\bar{x})$, since $f\left(x_{i}\right) \in L$, and since $L$ is closed, it follows that $f(\bar{x}) \in L$. Thus the proof of Theorem 4 has been completed.

3. Generalizations. The functions studied by $\mathrm{McShane}^{6}$ can be easily extended by the method of the preceding section. Let the onedimensional real-valued function $f(x)$ be defined over a set $S$ of a metric space. Furthermore suppose there exists a real-valued function $w(t) \geqq 0$ defined for $0 \leqq t$, and satisfying the conditions

$$
\begin{aligned}
w(a)+w(b) & \geqq w(a+b), \\
w(b) & \geqq w(a),
\end{aligned}
$$

for $b \geqq a$.

If the function $f(x)$ satisfies the condition

$$
\left|f\left(x_{1}\right)-f\left(x_{2}\right)\right| \leqq w\left(\| x_{1}, x_{2}||\right)
$$

for all pairs $\left(x_{1}, x_{2}\right)$ in $S$, then $f(x)$ can be extended to the entire space so as to preserve condition (22).

It should be noted that condition (20) does not imply that $w(t)$ be concave downward; however, any function $w(t) \geqq 0, t \geqq 0$, which is concave downward must satisfy condition (20). The function $w(t)$ is less restricted than that used by McShane. ${ }^{7}$

To prove the above theorem, let $S=\left(x_{1}, x_{2}\right)$ and suppose $x_{3}$ is a third point to which we wish to extend $f(x)$. With $f\left(x_{i}\right)(i=1,2)$ as centers draw circles $C_{i}^{\prime}$ with radii $r_{i}^{\prime} \equiv w\left(\left\|x_{i}, x_{3}\right\|\right)$, respectively. These circles are linear intervals. Suppose $C_{1}^{\prime} \cdot C_{2}^{\prime}=0$. Then by (20) and (21) we would have

$$
\begin{aligned}
\left|f\left(x_{1}\right)-f\left(x_{2}\right)\right| & >w\left(\left\|x_{1}, x_{3}\right\|\right)+w\left(\left\|x_{2}, x_{3}\right\|\right) \\
& \geqq w\left(\left\|x_{1}, x_{3}\right\|+\left\|x_{3}, x_{2}\right\|\right) \geqq w\left(\left\|x_{1}, x_{2}\right\|\right),
\end{aligned}
$$

${ }^{5}$ Loc. cit.

${ }^{6}$ Loc. cit.

7 Ibid. 
which contradicts the hypothesis $\left|f\left(x_{1}\right)-f\left(x_{2}\right)\right| \leqq w\left(\left\|x_{1}, x_{2}\right\|\right)$. Thus $C_{1}^{\prime} \cdot C_{2}^{\prime} \neq 0$, and any point $x_{3}^{\prime} \in C_{1}^{\prime} \cdot C_{2}^{\prime}$ serves as an extension $f\left(x_{3}\right)$. In order to extend $f(x)$ from an arbitrary set $U$ on which (22) holds, to a set $U+x_{0}$, with $f\left(x_{i}\right)$ as centers, $x_{i} \in U$, draw circles $C_{i}^{\prime}$ with radii $r_{i}^{\prime} \equiv w\left(\left\|x_{i}, x_{0}\right\|\right)$, respectively. Since each pair of the circles $C_{i}^{\prime}$ intersect, Helly's $s^{8}$ theorem, with $n=1$, implies that $\prod C_{i}^{\prime} \neq 0$. This implies that $f(x)$ can be extended from $U$ to $U+x_{0}$ so as to preserve condition (22). The extension of $f(x)$ from $S$ to the whole space now follows by transfinite induction.

An advantage of the above procedure arises from the fact that if the extension were impossible, the method would reveal it. Counterexamples exist which show that if $f(x)$ has its values in a metric space, then, in general, $f(x)$ cannot be extended so as to preserve conditions (1) and (22). For example, let $x$ range over a two-dimensional metric space in which all the "unit spheres" are congruent squares having corresponding sides parallel. Let $f(x)$ have its values in the two-dimensional Euclidean space. In Theorem 1, let. $C_{i}$ $(i=1,2,3)$ consist of three unit squares such that $C_{1} \cdot C_{2} \cdot C_{3} \equiv p$ consists of one and only one vertex from each square. Also choose $C_{i}^{\prime}$ so that $r_{i}^{\prime}=r_{i}=1, x_{1}^{\prime}=(1,0), x_{2}^{\prime}=(-1,0), x_{3}^{\prime}=(0,1+\epsilon), \epsilon>0$. The constant $\epsilon$ can be chosen so that condition (2) holds. Although condition (3) holds, conclusion (4) fails. Hence one cannot extend $f(x)$ from $S=\left(x_{1}, x_{2}, x_{3}\right)$ to $T=\left(x_{1}, x_{2}, x_{3}, p\right)$ so as to preserve condition (1).

The first generalization of the material in $\$ \S 1$ and 2 would be to the $n$-dimensional case, and thence to a Hilbert space. In the $n$-dimensional case the crux of this generalization lies in the generalization of Theorem 1, since that of Helly applies to the $n$-dimensional case. In the case of a Hilbert space one would also need a generalization of the theorem of Helly. These matters are still open questions. The author wishes to express his appreciation to Professor Max Zorn and to Dr. W. T. Puckett with whom he has had stimulating conversation concerning these topics.

University of California at Los ANgeles

${ }^{8}$ Loc. cit. 